\title{
"CUIDADOS POPULARES MEDICALIZADOS, CUIDADOS BIOMÉDICOS POPULARIZADOS. LA INCLUSIÓN DE LAS PARTERAS TRADICIONALES EN EL SISTEMA DE SALUD DEL ORIENTE DE GUATEMALA"
}

\author{
Lorenzo Mariano Juárez \\ Enfermero. Antropólogo
}

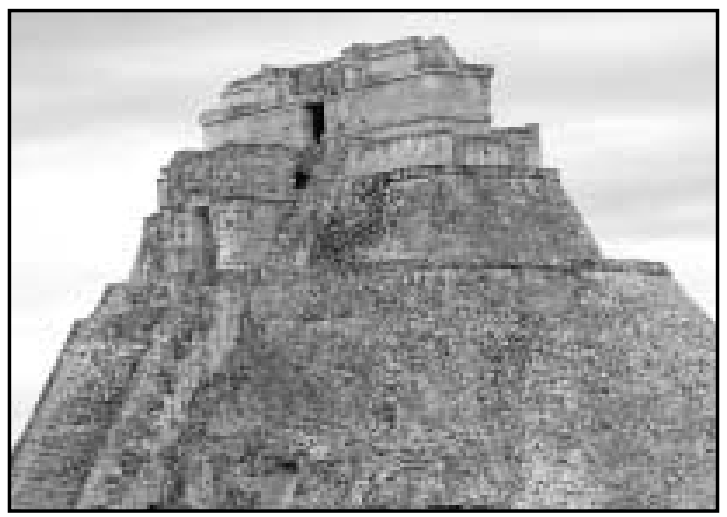

\section{SUMMARY}

Needs for increasing percentages of health care in Guatemala gave way to health decentralization in order to include ancient and traditional midwifes to cover and improve health participation in rural areas. Discussion on advantages and inconveniences of this fact are discussed here. There is a criticism on whether figures are real regarding bio-medical cover. The supposed to be community participation in the multi-cultural dayto-day care as is described here, implies not only a more than a simple process of medical education but various different ways of approaching understanding and caring for health problems and even understanding an assuming world in many ways. In many occasions figures stated here on education and consequent medical attention are far from real bio-medical attention patterns which seem to demonstrate such figures are nearer to a type of care where non-specialist and professional, popular and bio-medical attention overlap and mingle.

KEY WORDS: Bio-medical care, popular care, traditional midwifes, inter-cultural health, building health education concepts.

\section{RESUMEN}

La necesidad de ampliar los porcentajes de cobertura sanitaria en Guatemala ha motivado la descentralización sanitaria y la inclusión de las comadronas tradicionales y un fortalecimiento de la participación comunitaria en las zonas rurales. En el texto se discute sobre las ventajas e inconvenientes de este hecho y se critica la veracidad de las cifras de cobertura biomédica. Este fortalecimiento de la participación comunitaria en una realidad multicultural como la que se describe implica algo más que un simple proceso de formación médica, sino que envuelve aspectos tan singulares como formas diferentes de entender y atender la salud e incluso construir y entender el mundo. La realidad de esta formación y la consiguiente atención médica que se presta dista, en numerosas ocasiones, de los patrones de atención biomédica que parecen reflejar las cifras y se acerca más a un tipo de atención donde lo lego y lo profesional, lo popular y lo biomédico se solapan y confunden.

Palabras ClaVes: Cuidados biomédicos, cuidados populares. Parteras tradicionales. Salud intercultural. Construcción cultural de la salud.

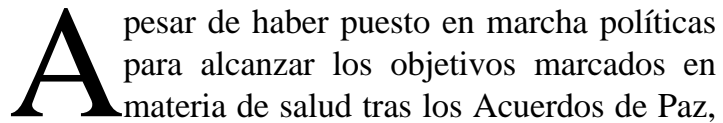
los actuales indicadores de salud son deficientes para toda Guatemala. El porcentaje de población que vive en la pobreza o en la extrema pobreza es uno de los mayores de todo el planeta, y las cifras son similares en cuanto a índices de desnutrición o de desigualdad'. A pesar de incrementar el gasto sanitario en los últimos años, se mantienen unas de las peores cifras de desempeño en el área de salud de toda América Latina. Las cifras empeoran entre los sectores rurales e indígenas y el porcentaje de 
población con acceso a la asistencia médica está lejos de lo deseable. A pesar de un crecimiento negativo de PIB por habitante, en los últimos años se han desarrollado programas como el SIAS (Sistema Integral de Atención en Salud), un sistema descentralizado que opera generalmente a través de Ongs o Cooperativas y el Plan Nacional de Salud 200-2004, que prioriza la atención médica primaria y la descentralización con la participación social y comunitaria.

Dentro de este programa se incluyeron en las zonas rurales a las comadronas tradicionales y la figura del promotor o guardián de salud, integrantes de la comunidad de referencia a los que se les capacita en salud bajo las directrices o postulados alopáticos. Se trata de un sistema jerarquizado que otorga presencia y cobertura sanitaria en todas las comunidades rurales, al menos respecto a las cifras. Este texto intenta ofrecer un discurso crítico hacia este modelo y desvelar la opacidad de algunas de esas cifras, mostrando como a pesar de que se habla de un aumento muy significativo de cobertura en salud, ello no significa un aumento significativo de cobertura biomédica.

Como comentaba, el SIAS es un sistema descentralizado en el que la atención primaria de salud en las comunidades es prestada por las comadronas tradicionales y por personas de la comunidad. El área de estudio es la comunidad de Jocotán, en el oriente del país, una zona pobre donde conviven ladinos e indígenas $\mathrm{C}$ 'hortí. La formación o la capacitación de las comadronas indígenas se realiza mediante una serie de cursos donde se ofrecen los conocimientos específicos que se recogen en un manual y que en teoría consiguen reciclar la atención en salud de estas comadronas. El procedimiento es similar para los guardianes de salud, salvo que éstos son elegidos en cada comunidad por sus propios vecinos. Posteriormente se realizan capacitaciones monográficas sobre diversos temas una o en ocasiones dos veces al mes. Este modelo de atención implica dificultades en la categorización tanto de comadronas como de guardianes de salud. Teóricamente se trata de un fomento de la participación ciudadana, pero estas figuras gozan de un estatus alejado de lo profano o lo lego; a efectos clasificatorios y estadísticos, son parte integrante de pleno derecho de la atención biomédica y en teoría la primera e indispensable forma de cuidado y atención biomédico. Aunque la práctica y experiencia diaria dista un poco de lo descrito.

Recuerdo una de las capacitaciones mensuales a las comadronas del área. La sesión se había dividido en dos días, para un trato más personalizado, de forma que en cada sesión había cerca de treinta comadronas capacitadas. El tema de la sesión se centraba en el modo de enseñar a las madres la forma correcta de realizar la lactancia. La sesión estaba preparada con un video de apenas 10 minutos de duración en el que se hacía hincapié en los aspectos esenciales de la lactancia materna. A continuación debería seguir una mesa redonda donde se comentara lo que se había visto y las diferencias que encontraban con sus conocimientos o su práctica. A pesar de los ingentes esfuerzos del médico que dirigía la capacitación por realizar un discurso intercultural que se alejase de postulados netamente hegemónicos y absolutos e intentando subrayar que los conocimientos no debieran chocar con lo que ya sabían las comadronas, creo que los resultados de la capacitación fueron nulos: el video se prolongó cerca de la hora, con constantes paradas y repeticiones para explicar el significado de nuevos términos, como areola o mama. Las caras de las comadronas oscilaban entre la indiferencia y el sopor. Al final, la mayoría de ellas se marcharon contentas por los nuevos conocimientos que habían adquirido: ya sabían que significaban palabras como las que he nombrado. No sé si incluirían este nuevo vocabulario en su educación a las madres, pero parece claro que ninguna reparó en la forma adecuada de dar de mamar.

Este hecho puede observarse solo bajo una perspectiva anecdótica o incluso como un evidente fallo de organización de la capacitación intercultural, pero sirve para lo que pretendo argumentar. Evidentemente, la formación biomédica implica conocer un nuevo lenguaje y quizá ello se trate de un hecho imprescindible. Pero el proceso va más allá. Convertir los cuidados profanos en cuidados profesionales implica adentrarse en una nueva forma de ver y concebir el mundo. Como señala Good (2003:145), "aprender el lenguaje de la medicina no consiste en aprender nuevas palabras para denotar el mundo corriente, sino construir un mundo completamente nuevo". Si este salto es con- 


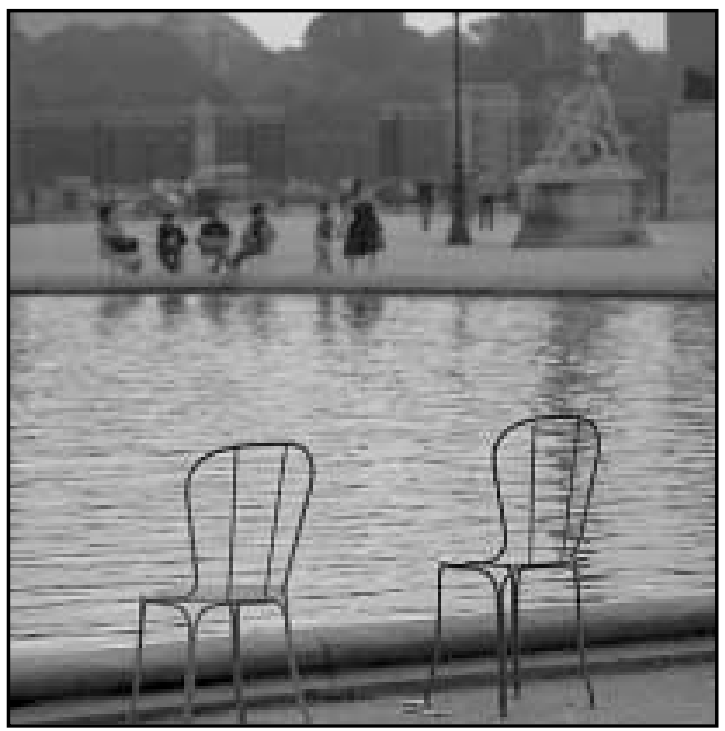

siderable dentro de un mismo grupo cultural, lo es aún más cuando se trata de culturas diferentes. En esta ocasión, lo que se pretende es convertir un cuidador profesional (comadronas dentro de su cultura) en un cuidador profesional con un concepto distinto de la salud.

Sin embargo sabemos que la salud es un término culturalmente relativo, en el que el ejercicio diagnóstico se acerca a un proceso cultural que permite construir las enfermedades más que descubrir$\operatorname{las}^{2}$ (Gaines, 1985) y en un contexto donde la enfermedad es definida y tipificada por cada cultura, que la dota de significación social (Salas Iglesias, 2001:26). Los procesos de capacitación en salud a comadronas y guardianes de salud no pueden entenderse como clases de baile en donde cada día se dominan más pasos. Lo aprendido sumerge a los actores sociales en un mundo completamente nuevo; los procedimientos asistenciales y las categorizaciones son fruto de la imbricación de todo lo que se sabía y lo que ahora se aprende. En este proceso, lo popular y lo biomédico se funden en nuevas formas de atención.

A pesar de que las comadronas se identifiquen con un nuevo estatus al verse capacitadas, que porten mochilas con nuevos y médicos instrumentos, lo cierto es que sus prácticas no se ajustan a la atención biomédica; dependiendo de muy diferentes factores, se deciden por las prácticas tradicionales como soplar tabaco o pasar hoja de ruda como se puedan mostrar proclives a la vacunación o a la anticoncepción. En este sentido las posibilidades son numerosas y los pasos del tango se confunden con los del vals. Pero la forma de entender la salud, de construir la enfermedad y el padecimiento se encuentra sometida a disputas personales entre la tradición y la biomedicina. El resultado es una mezcla de saberes, prácticas y representaciones en las que es difícil discernir entre lo hegemónico y lo subalterno y entre lo profano y lo profesional.

Durante el trabajo de campo en la zona trabajamos con la construcción local del hambre y la desnutrición. De alguna manera, son conceptos que carecen de una relación directa en la ideología C’hortí, de forma que la etiología de la desnutrición opera en una compleja red de significación y comportamientos que se alejan de la carencia de alimentos. A pesar de las intensas capacitaciones en la zona $^{3}$, la construcción social de la desnutrición se mantiene alejada de la idea biomédica. Aunque esto es perfectamente esperable para la población profana, parece lógico esperar que tanto los guardianes de salud como las comadronas se posicionen dentro de la construcción de la salud y las formas de atendimiento de la atención alopática. Aunque esto no siempre sucede. Dentro de la atención a la desnutrición como padecimiento, especialmente en la edad infantil, una de las primeras divergencias tiene que ver con la categorización de síntomas como desnutrición. En el ideario local el marasmo es identificado como delgadito, sequito, disnutrío. Sin embargo, la imagen del kwashiorkor remite a otra categorización, como lombrices, enfermo sin definir o en ocasiones la categoría alentadito, que aleja la situación de lo patológico. Esto en principio no parece algo inusual, pues de alguna manera puede entenderse que la carencia lleva a estados carenciales distantes de la imagen del Kwashiorkor.

Una recapitulación de las entrevistas en profundidad realizadas arroja cifras muy parejas en la identificación de la imagen del marasmo: 31 de los 37 entrevistados del grupo de cuidados profanos consideró la imagen como desnutrición, frente a los 24 de 28 entre los cuidadores profesionales. Respecto a la imagen del Kwashiorkor, el cuidado lego tiende a identificarlo como un estado patológico (25 de 37) y nadie lo identificó como desnutrido. En el caso de las comadronas y los guardianes de 


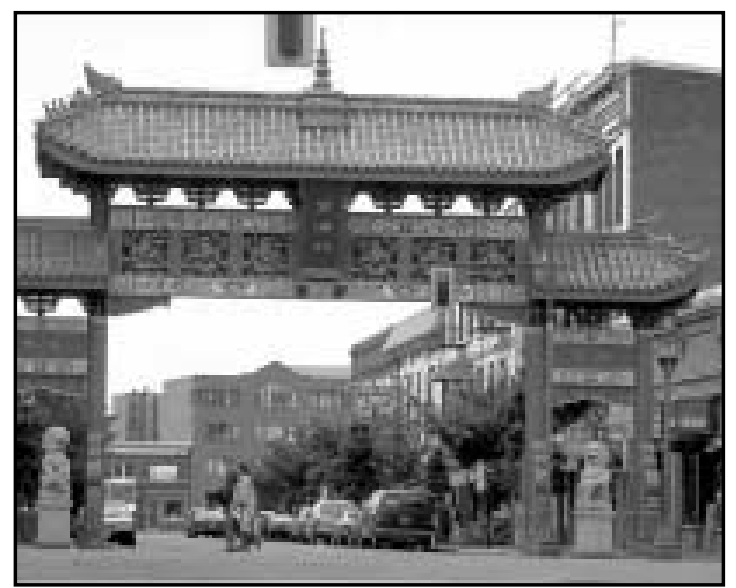

salud, solo 4 de las 28 entrevistas se decantaron por definirlo como desnutrido, a pesar de que la lámina utilizada en la investigación era la misma que la que se usa en las capacitaciones y que puede verse en muchas de las casas de todas las comunidades.

Sin embargo, este tipo de análisis se encuadran dentro de los problemas interculturales que nacen de la categorización, es decir, de diferentes procesos de designación relativos culturalmente sobre hechos idénticos, ajenos a la cultura, sobre los que existe abundante literatura (por ejemplo Kortmann, 1990, Abad,1979) y que son "fáciles de imbricar en la "hermenéutica biomédica" (Good, 2003:175). Desde esta perspectiva superficial, el análisis resulta incompleto y los obstáculos a la medicalización de las prácticas de las comadronas y guardianes de salud fácilmente superables. Pero un análisis más profundo muestra la complejidad en la que operan los procesos de construcción cultural de la salud y la dificultad de que el cambio opere en ellos.

Lo cierto es que la identificación del marasmo en el ideario local como disnutrío se aleja de la categoría "desnutrido" biomédico. Se trata de un padecimiento que tiene su particular etiología ${ }^{4}$ en la que la carencia de alimentación no es el eje principal y este trastorno se encuadra en una compleja red de significados, estructuras y relaciones de poder. Es en esta concepción donde más debería notarse los efectos de las capacitaciones, pero las ideas en torno a la etiología de la desnutrición ofrecen una situación similar en los discursos del cuidado lego y las comadronas y los guardianes de salud. En alguna ocasión los discursos de éstos se acercan a los postulados biomédicos, aunque se aprecia una carencia en la adquisición de ese lenguaje médico, y se trata de causas que de alguna manera son compartidas por los dos sistemas de atención. Así se refería una comadrona a la etiología de la desnutrición infantil:

"Ese está enfermo, digamos, este niño está bien desnutrido, por falta de atendimiento de papá y mamá, si porque la mamá estuvo muy, muy... si porque la mamá hay veces que a los seis meses que tiene... y ya el otro ya está guardao ya en el vientre siempre porque hay mamás que muy luego tiene el niño al año está el otro, ¿por qué?, porque a los seis meses el otro ya está en barriga. Entonces por eso al nacer este niño, siempre el niño que está ya nacido, ya vuelve a quedarse sin amamantar, ya no mama pecho, solo le dan seis mese de leche y después ya le quitan el pecho, entonces ya no le dan, le dan comida, tortilla, tal vez con frijol y tal vez el niño todavía el estómago no... no, no, todavía no muele, no tiene dientadura, digamos el, el corazón, porque tiene una maquinita donde trabaja, y todavía ese no, no mastica bien entonces el niño mejor se enferma. Todavía los pulmones no tienen venas fuertes para mandar esa sangre en los pulmones..."

Pero en la mayoría de ocasiones la distancia es muy grande entre el conocimiento de las comadronas y la práctica biomédica y entre la etiología se mezclan antojos no consentidos, sustos o incluso relaciones sexuales con maridos ebrios. En la mayoría de las ocasiones, los discursos de las comadronas apenas se diferencian del cuidado lego, aunque a veces se hace referencia a que lo que nos cuentan es un discurso aprendido en las capacitaciones:

"se viene como que algo impedido, está tullido... y una parte como dice el decir que una criatura se tulle en vientre de la mamá porque puede ser que algún espanto tiene la mamá, eso es lo que tiene... habemos mucho con eso en decir que si uno le atina un buen espanto, como es fue de espanto junto al fuego, espanto la claridad de San Antonio, así quedan los chiquitos, porque yo también..., yo ya les dicen varios que las criatura vienen impedidos, porque impedimiento no, porque más que el agarramiento que lo agarra onde uno está cocineando, es que como dice el decir, nadie... en San 
Antonio servicial, los da de comer y los hace las cosas, los da pero no sabemos como jugan allí por una mujer interesa... ese está también como... vaya como le digo yo que tal vez una enfermedad le tuvo esa criatura, que siempre allí le cayó... también una parte puede ser que es caído junto al fuego, porque ya desde lejos se quedó así quizás" Comadrona

“... enfermito, porque... este que nació así, desde que nació así... por eso muchos están así onde estamos, en Yagualá ... hay un muchachito primerito, mi sobrino, así como ese, ya tiene ocho años..... ¿Usted no sabe por qué será? Porque dicen que bolo, bolo el hombre se... se, sostientan las mujeres bolos, por eso todos chemblejos salen, así dicen, así nos predican ustedes, onde nosotros vamos nos dicen". Comadrona

Este paralelismo en los discursos profanos y profesionales sobre la etiología se producen también en la prevención o la terapéutica, aunque existen diferencias significativas por edad, género o comunidad en la que trabajen. Lo profesional entendido bajo lo biomédico se difumina en este tipo de atención médica, que se aleja de los protocolos y conocimientos alopáticos. Las clásicas recomendaciones de Paul (1955:1) para aprender a pensar como los miembros de una comunidad cuando deseemos mejorar su salud no equivale a la "contratación" de comadronas o guardianes de salud y de alguna manera surge un problema similar cuando éstos tienen que aprender a pensar como nosotros.

\section{CONCLUSIONES}

A la vista de lo señalado podemos realizar una recapitulación a modo de conclusiones. La primera de ellas tiene que ver con el tipo de atención en salud prestada por los guardianes de salud y las comadronas. A pesar de que las cifras de cobertura han aumentado con la descentralización de los servicios de salud y la inclusión de estos agentes, queda claro que no se trata de una atención que se acoja a los postulados biomédicos. La opacidad de esas cifras no permite desvelar la complejidad de una realidad en la que se entremezclan prácticas tradicionales con formas biomédicas de atención en salud. En segundo lugar, creo que es necesario no subestimar los problemas de traducción y diálogo intercultural en salud; me opongo a una mitificación de estas realidades, pero es necesario afrontar estos contextos más allá de la problemática de categorización y adentrarnos en una perspectiva relacional y simbólica de construcción del padecimiento. Si reconocemos que la forma diferente de construir modelos de salud y enfermedad va más allá de denominaciones diferentes sino que incluye modos diversos de entender y habitar el mundo somos capaces de vislumbrar las deficiencias de este modelo descentralizado.

Por último, creo que es necesario recordar que "las decisiones sobre la salud están mucho más constreñidas por factores sociales objetivos $\mathrm{y}$ macroestructuras de desigualdad, según aducen muchos, que por creencias subjetivas o factores cognitivos" (B. Good, 2003:93). Esto ha dado pie a debates que enfrentan el papel de la creencia y la estructura, como la disponibilidad o el coste de tratamiento (J. Young, 1981). En el texto se ha defendido una crítica a este modelo descentralizado basado en teorizaciones y presupuestos bajo un marco cognitivo-construccionista de la salud. Pero creo necesario defender que este discurso está producido por diferentes estructuras de poder y situaciones de desigualdad de género o etnia. La perspectiva relacional debe incluir un análisis de esas variables estructurales y el papel que juegan en la "producción social de la salud”. Ese análisis debería ofrecer respuestas a los motivos de descentralización del sistema de salud en las zonas rurales de población indígena y la ampliación de cobertura alopática sin aumentar notablemente médicos y enfermeras que vayan más allá de la falta de medios económicos. Las variables cognitivas son un elemento esencial de análisis que puede condicionar la aplicación de un modelo concreto como hemos señalado en el texto, pero no puede obviarse un análisis que entienda "las cuestiones de la salud a la luz de las fuerzas políticas y económicas más amplias que modelan las relaciones interpersonales, forman comportamiento social, generan significados sociales y condicionan la experiencia colectiva"(Singer, 1990:181). Este modelo descentralizado defiende la tesis del mínimo presupuestario, pero es necesario cuestionarse ese modelo y las causas que abocan a él desde una perspectiva global, además de los obstáculos que surgen de la perspectiva cognitiva. 


\section{BIBLIOGRAFÍA}

- ABAD, B. y Boyce, E. (1979) "Issues in Psychiatric Evaluation of Puerto Ricans: a Sociocultural Perspective". Journal of Operational Psychiatry. 10, 28 39.

- ÁlVAREZ ARAGÓN, V. (Comp) (2003); El rostro indígena de la pobreza. Guatemala, Flacso.

- ARRIZABALGA, J., (2000) "Cultura e Historia de de la Enfermedad", en Medicina y Cultura. Estudios entre la antropología y la medicina. Perdiguero E., Comelles, J.M. (eds). Ed. Bellaterra, Barcelona.

- GOOD, B. J. (2003) "Medicina, racionalidad y experiencia. Una perspectiva antropológica,” Ed. Bellaterra, Barcelona.

- GAINES, A. D., y HAHN, R. (1985) "Among the Physicians: Encounter, Exchange and Transformation", En Physicians of Western Medicine. Anthropological Approaches to Theory and Practice. Gaines, A. D., Hahn R (Eds.) Dordrecht, Reidel: 3-22.

- KORTMANN, F. (1990) "Psychiatric Case Finding in Ethiopia: Shortcomings of the Self Reporting Questionnaire". Culture, Medicine and Psychiatry, 14 :381-391

- Paul, B. D. (1955) Health, Culture and Community: Case Studies of Public Reactions to Health Programs, Rusell Sage Foundation, Nueva York

- SALAS IGLESIAS M. J. (2001) "Terapias alternativas". En Index de Enfermería, Granada, 35: 25-28.

- SINGER M. (1990) "Reinventing Medical Anthropology: Toward a Critical Realignment", en social Science and Medicine, 30. 79-187

- YOUNG, J. (1981) "Medical Choice in a Mexican Village, Rutgers University Press, New Brunswick.

\section{NOTAS}

${ }^{1}$ Véanse los informes del LCSHD, del Banco Mundial, La pobreza en Guatemala, informe 24221-GU. La situación es consecuencia de los procesos de exclusión y desigualdades existentes, motivadas por factores culturales, niveles de asistencia sanitaria o grados de formación educativa. Aunque las cifras varían en función de los estudios y las etnias, se acercan al $90 \%$ de indígenas que viven por debajo de la línea de la pobreza. (Álvarez Aragón, 2003). el último informe del Banco Mundial señala a Guatemala como una de las regiones con lo índices de pobreza más altos de la zona y alcanza el primer puesto en índices de desnutrición. Por ejemplo, el índice de Gini para consumo e ingreso es de 48 y 57 respectivamente. A pesar de que la pobreza parece haber disminuido en los últimos años alcanza cifras escandalosas. Los índices de desnutrición también disminuyen, aunque menos que en otras regiones del área; el crecimiento infantil muestra tasas de crecimiento insuficiente cercanas al $50 \%$
2 Las aproximaciones constructivistas o socioconstructivistas han acabado por impregnar en mayor o menor medida una gran parte de los estudios históricos y antropológicos sobre la enfermedad. Véase Arrizabalaga, (2000:71-82), para un repaso histórico.

${ }^{3}$ Especialmente a partir de la hambruna del 2001, donde la zona acaparó las portadas de los medios de comunicación de todo el mundo ante la alarmante escasez alimentaria y que supuso enormes críticas para la gestión de Gobierno de----1 presidente Portillo.

${ }^{4}$ Aunque merecería un análisis particular, las causas de la desnutrición infantil se agrupan en diversas prácticas en las que la responsabilidad recae en las madres: no les dan de comer a las horas, se quedan embarazadas demasiado pronto, no atendieron algún capricho, despistarse en otros quehaceres y dejar al niño mucho tiempo en el suelo, no evitar que coma tierra, darle alimentos que no le toman sabor o tenerlo sucio entra dentro de la falta de cuidado. La falta de alimentos para ofrecerle al niño rara vez es esgrimido en los discursos. 